T. Konno and S. Tanno

Nagoya Math. J.

Vol. 151 (1998), 91-97

\title{
GEODESICS AND KILLING VECTOR FIELDS ON THE TANGENT SPHERE BUNDLE
}

\author{
TATSUO KONNO AND SHUKICHI TANNO
}

\begin{abstract}
We show that any Killing vector field on the unit tangent sphere bundle with Sasaki metric of a space of constant curvature $k \neq 1$ is fiber preserving by studying some property of geodesics on the bundle. As a consequence, any Killing vector field on the unit tangent sphere bundle of a space of constant curvature $k \neq 1$ can be extended to a Killing vector field on the tangent bundle.
\end{abstract}

\section{$\S 1$. Introduction}

Let $\left(T_{1} M, g^{S}\right)$ be the unit tangent sphere bundle with Sasaki metric $g^{S}$ of a Riemannian manifold $(M, g)$. A Killing vector field $Z$ on $\left(T_{1} M, g^{S}\right)$ is called fiber preserving if each (local) isometry $\phi_{t}$ generated by $Z$ maps each fiber into a fiber. If $(M(k), g)$ is a space of constant curvature $k$, then geodesic flow vector $\xi$ of $\left(T_{1} M(k), g^{S}\right)$ is a Killing vector field if and only if $k=1$ (cf. Tanno [6]). It is important to notice that $\xi$ is not fiber preserving.

In this paper we obtain the following:

THEOREM A. Let $\left(T_{1} M(k), g^{S}\right)$ be the unit tangent sphere bundle of a space $(M(k), g)$ of constant curvature $k$. If $k \neq 1$, then any Killing vector field on $\left(T_{1} M(k), g^{S}\right)$ is fiber preserving.

One of the authors [3] proved that any Killing vector field $Z$ on the unit tangent sphere bundle $\left(T_{1} M, g^{S}\right)$ of a Riemannian manifold $(M, g)$ can be extended to a Killing vector field on the tangent bundle $\left(T M, g^{S}\right)$ if $Z$ is fiber preserving. So, by Theorem A we have the following:

THEOREM B. Any Killing vector field on the unit tangent sphere bundle $\left(T_{1} M(k), g^{S}\right)$ of a space $(M(k), g)$ of constant curvature $k \neq 1$ can be extended to a Killing vector field on the tangent bundle $\left(T M(k), g^{S}\right)$.

To prove Theorem A we study some property of geodesics on $\left(T_{1} M(k)\right.$, $\left.g^{S}\right)$ in $\S 2$. As for basic geometry on the tangent bundle or tangent sphere bundle, cf. Dombrowski [1], Sasaki [5], etc.

Received March 21, 1997. 
§2. Geodesics in $\left(T_{1} M(k), g^{S}\right)$

Let $\left(T_{1} M, g^{S}\right)$ be the unit tangent sphere bundle with Sasaki metric $g^{S}$ of a Riemannian manifold $(M, g)$. By $\pi$ we denote the projection from $T_{1} M$ to $M$. Let $\tilde{C}=\{(x(\sigma), y(\sigma)) ; 0 \leq \sigma \leq l\}$ be a curve in $\left(T_{1} M, g^{S}\right)$ with arc-length parameter $\sigma$, where $y(\sigma) \in T_{x(\sigma)} M, g(y(\sigma), y(\sigma))=1$. It is a geodesic if and only if

$$
\begin{aligned}
& \left(\nabla_{x^{\prime}} x^{\prime}\right)(\sigma)=-R\left(y(\sigma),\left(\nabla_{x^{\prime}} y\right)(\sigma)\right) x^{\prime}(\sigma) \\
& \left(\nabla_{x^{\prime}} \nabla_{x^{\prime}} y\right)(\sigma)=\rho(\sigma) y(\sigma)
\end{aligned}
$$

hold for some function $\rho(\sigma)$, where $\nabla(R$, resp.) denotes the Riemannian connection (Riemannian curvature tensor, resp.) of $(M, g)$, and $x^{\prime}(\sigma)=$ $d x(\sigma) / d \sigma$ (cf. Sasaki [5], II, p.152).

Geodesics on the unit tangent sphere bundle $\left(T_{1} S^{2}(1), g^{S}\right)$ of a unit 2-sphere $\left(S^{2}(1), g\right)$ were studied by Klingenberg and Sasaki [2]. For a unit $m$-sphere $\left(S^{m}(k), g\right), m \geq 3$, and for more general $(M(k), g)$, we have the following (cf. Nagy [4]):

THEOREM C. Let $\left(T_{1} M(k), g^{S}\right)$ be the unit tangent sphere bundle of a space $(M(k), g)$ of constant curvature $k$, and let $\tilde{C}=\{(x(\sigma), y(\sigma)) ; 0 \leq$ $\sigma \leq l\}$ be a geodesic with arc-length parameter $\sigma$ in $\left(T_{1} M(k), g^{S}\right)$. By $C=\{x(\sigma)\}$ we denote the projection $\pi \tilde{C}$ of $\tilde{C}$. Then $\left\|x^{\prime}\right\|^{2}=1-c^{2}$ is constant, where $0 \leq|c| \leq 1$.

(i) If $|c|=1$, i.e., $C$ reduces to a point, then $\tilde{C}$ is a (piece of) great circle in a fiber and $y$ is rotated in a 2-plane at $x(0)$.

(ii) If $0<|c|<1$, then we have the following:

(ii-a-1) The geodesic curvature $\kappa$ of $C$ is constant.

(ii-a-2) C satisfies

$$
\nabla_{x^{\prime}} \nabla_{x^{\prime}} \nabla_{x^{\prime}} x^{\prime}=-k^{2} c^{2} \nabla_{x^{\prime}} x^{\prime}
$$

(ii-b-1) If $k=0$, then $\kappa=0$ and we have parallel orthonormal vector fields $\left\{E_{1}, E_{2}\right\}$ along $C$ such that

$$
y(\sigma)=\cos c \sigma \cdot E_{1}(\sigma)+\sin c \sigma \cdot E_{2}(\sigma) .
$$

(ii-b-2) If $k \neq 0$ and $\kappa=0$, then we have parallel orthonormal vector fields $\left\{E_{1}, E_{2}, x^{\prime} /\left\|x^{\prime}\right\|\right\}$ along $C$ such that $y$ is of the form (2.3). 
(ii-b-3) If $k \neq 0$ and $\kappa \neq 0$, then we have parallel orthonormal vector fields $\left\{E_{1}, E_{2}\right\}$ along $C$ such that $y$ is of the form (2.3).

(ii -c) The angle $\theta(\sigma)$ between $y(\sigma)$ and $x^{\prime}(\sigma)$ is given by

$$
\cos \theta(\sigma)=\left[\alpha / \sqrt{1-c^{2}}\right] \sin [(1-k) c \sigma+\beta]
$$

where $\alpha$ and $\beta$ are constant. $\alpha^{2}$ is given by (2.9) and $\alpha=0$ for (ii-b-2).

(iii) If $c=0$, then $C=\{x(\sigma)\}$ is a geodesic with arc-length parameter $\sigma$, and $y$ is a parallel vector field along $C$.

Proof. By (2.1) the equations of geodesic in $\left(T_{1} M(k), g^{S}\right)$ is given by

$$
\nabla_{x^{\prime}} x^{\prime}=-k b y+k a \nabla_{x^{\prime}} y, \quad \nabla_{x^{\prime}} \nabla_{x^{\prime}} y=\rho y
$$

where we have put

$$
a=a(\sigma)=g\left(x^{\prime}, y\right), \quad b=b(\sigma)=g\left(x^{\prime}, \nabla_{x^{\prime}} y\right)
$$

and we sometimes omit the parameter $\sigma$ from the expression for simplicity. We put

$$
c^{2}=c^{2}(\sigma)=g\left(\nabla_{x^{\prime}} y, \nabla_{x^{\prime}} y\right)
$$

By $g(y, y)=1$, we have $g\left(y, \nabla_{x^{\prime}} y\right)=0$ and

$$
g\left(\nabla_{x^{\prime}} y, \nabla_{x^{\prime}} y\right)+g\left(y, \nabla_{x^{\prime}} \nabla_{x^{\prime}} y\right)=0, \quad \text { i.e., } \quad c^{2}+\rho=0 .
$$

Differentiating (2.7) and using $(2.5)_{2}$ we see that $c$ is constant.

Let $X$ be a tangent vector at a point of $(M(k), g)$. By $X^{H}$ or $X^{V}$ we denote the horizontal lift or vertical lift of $X$ to $\left(T M(k), g^{S}\right)$ or $\left(T_{1} M(k), g^{S}\right)$. Since the tangent vector field $T$ of $\tilde{C}$ is expressed as

$$
T=\left(\frac{d x}{d \sigma}, \frac{d y}{d \sigma}\right)=x^{\prime H}+\left(\nabla_{x^{\prime}} y\right)^{V}
$$

we have $1=\|T\|^{2}=\left\|x^{\prime}\right\|^{2}+c^{2}$. Therefore $\left\|x^{\prime}\right\|^{2}=1-c^{2}$ is constant, and the parameter $\sigma$ of $C=\{x(\sigma)\}$ is proportional to the arc-length.

If $|c|=1$, i.e., $\left\|x^{\prime}\right\|=0$, then $\tilde{C}$ is a geodesic in a fiber. Since each fiber is totally geodesic and isometric to a unit $(m-1)$-sphere, it is a (piece of) great circle. So, $y$ is expressed as $y(\sigma)=\cos \sigma \cdot e_{1}+\sin \sigma \cdot e_{2}$ for some orthonormal vectors $\left\{e_{1}, e_{2}\right\}$ at $x(0)$. 
Next we assume $0<|c|<1$. Calculating $a^{\prime}=\nabla_{x^{\prime}} a$ and $b^{\prime}=\nabla_{x^{\prime}} b$, we obtain

$$
a^{\prime}=(1-k) b, \quad b^{\prime}=-(1-k) a c^{2} .
$$

Operating $\nabla_{x^{\prime}}$ to $(2.5)_{1}$ twice, we obtain $(2.2)$. By $(2.5)_{1}$ we obtain

$$
\left\|\nabla_{x^{\prime}} x^{\prime}\right\|^{2}=k^{2}\left(c^{2} a^{2}+b^{2}\right)
$$

By (2.8) we can show that $c^{2} a^{2}+b^{2}$ is constant, and the geodesic curvature $\kappa$

$$
\kappa^{2}=k^{2}\left(c^{2} a^{2}+b^{2}\right) /\left(1-c^{2}\right)^{2} .
$$

of $C$ is constant. This proves (ii-a-1) and the first part of (ii-b-1). By (2.8) again, we have

$$
a(\sigma)=\alpha \sin [(1-k) c \sigma+\beta], \quad b(\sigma)=c \alpha \cos [(1-k) c \sigma+\beta]
$$

where $\alpha$ and $\beta$ are constant. Here, $\alpha^{2}$ is expressed as

$$
\alpha^{2}=\left(c^{2} a^{2}+b^{2}\right) / c^{2} \quad\left(\alpha^{2}=\left(1-c^{2}\right)^{2} \kappa^{2} / c^{2} k^{2}, \text { if } k \neq 0\right)
$$

The angle $\theta(\sigma)$ between $y(\sigma)$ and $x^{\prime}(\sigma)$ is given by $\cos \theta(\sigma)=g\left(x^{\prime} /\left\|x^{\prime}\right\|\right.$, $y)=a(\sigma) / \sqrt{1-c^{2}}$ and we obtain (2.4).

Now we define vector fields $E_{1}$ and $E_{2}$ along $C$ by

$$
\begin{aligned}
& E_{1}(\sigma)=\cos c \sigma \cdot y(\sigma)-\sin c \sigma \cdot\left(\nabla_{x^{\prime}} y\right)(\sigma) / c \\
& E_{2}(\sigma)=\sin c \sigma \cdot y(\sigma)+\cos c \sigma \cdot\left(\nabla_{x^{\prime}} y\right)(\sigma) / c
\end{aligned}
$$

Then $E_{1}$ and $E_{2}$ are parallel orthonormal vector fields along $C$, and define a parallel 2-plane field $\Pi$ along $C . y$ is rotated in $\Pi$ as

$$
y(\sigma)=\cos c \sigma \cdot E_{1}(\sigma)+\sin c \sigma \cdot E_{2}(\sigma)
$$

This proves (ii-b-1) and (ii-b-3). If $k \neq 0$ and $\kappa=0$, then $a=b=0$. So, $\left\{E_{1}, E_{2}, x^{\prime} /\left\|x^{\prime}\right\|\right\}$ are orthonormal and we have (ii-b-2).

Finally, if $c=0$, then we have $\nabla_{x^{\prime}} y=0, \nabla_{x^{\prime}} x^{\prime}=0$ and (iii). 


\section{$\S 3$. The converse of Theorem $\mathbf{C}$}

TheOREM D. Let $(M(k), g)$ be a space of constant curvature $k$. The converse of two cases (i) and (iii) of Theorem $C$ is trivial. So, let $C=$ $\{x(\sigma) ; 0 \leq \sigma \leq l\}$ be a curve of constant geodesic curvature $\kappa$ with $\left\|x^{\prime}\right\|^{2}=$ $1-c^{2}, 0<|c|<1$. Assume that $C$ satisfies

$$
\nabla_{x^{\prime}} \nabla_{x^{\prime}} \nabla_{x^{\prime}} x^{\prime}=-k^{2} c^{2} \nabla_{x^{\prime}} x^{\prime}
$$

(ii*-1) If $k=0$, then we assume $\kappa=0$. Let $\left\{E_{1}, E_{2}\right\}$ be parallel orthonormal vector fields along $C$ and define a vector field $y$ along $C$ by

$$
y(\sigma)=\cos c \sigma \cdot E_{1}(\sigma)+\sin c \sigma \cdot E_{2}(\sigma) .
$$

Then $\tilde{C}=\{(x(\sigma), y(\sigma))\}$ is a geodesic in $\left(T_{1} M(k), g^{S}\right)$.

(ii*-2) If $k \neq 0$ and $\kappa=0$, then let $\left\{E_{1}, E_{2}, x^{\prime} /\left\|x^{\prime}\right\|\right\}$ be parallel orthonormal vector fields along $C$. Define y by (3.2). Then $\tilde{C}=\{(x(\sigma), y(\sigma))\}$ is a geodesic in $\left(T_{1} M(k), g^{S}\right)$.

(ii*-3) If $k \neq 0$ and $\kappa \neq 0$, then let $e_{1}=\left(\nabla_{x^{\prime}} x^{\prime}\right)(0) /\left(1-c^{2}\right) \kappa$ and

$$
e_{2}=\left(\nabla_{x^{\prime}} \nabla_{x^{\prime}} x^{\prime}\right)(0) / k c\left(1-c^{2}\right) \kappa .
$$

Define $\left\{E_{1}, E_{2}\right\}$ along $C$ by parallel translation of $e_{1}$ and $e_{2}$. Next we define $y$ by

$$
y(\sigma)=\cos (c \sigma+\gamma) \cdot E_{1}(\sigma)+\sin (c \sigma+\gamma) \cdot E_{2}(\sigma)
$$

for constant $\gamma$. Then $\tilde{C}=\{(x(\sigma), y(\sigma))\}$ is a geodesic in $\left(T_{1} M(k), g^{S}\right)$.

Proof. First we prove (ii $\left.{ }^{*}-1\right)$ and (ii -2$)$. By $\kappa=0$ we have $\nabla_{x^{\prime}} x^{\prime}=0$. By (3.2) we obtain $\nabla_{x^{\prime}} \nabla_{x^{\prime}} y=-c^{2} y$ and, using $g\left(x^{\prime}, y\right)=g\left(x^{\prime}, \nabla_{x^{\prime}} y\right)=0$ for (ii*-2), we have (2.5).

Next, we show (ii*-3). Since $\nabla_{x^{\prime}} x^{\prime} /\left(1-c^{2}\right) \kappa$ is a unit vector field along $C$, we see that $\nabla_{x^{\prime}} x^{\prime}$ and $\nabla_{x^{\prime}} \nabla_{x^{\prime}} x^{\prime}$ are orthogonal. Using (3.1), we obtain

$$
g\left(\nabla_{x^{\prime}} \nabla_{x^{\prime}} x^{\prime}, \nabla_{x^{\prime}} \nabla_{x^{\prime}} x^{\prime}\right)=k^{2} c^{2} g\left(\nabla_{x^{\prime}} x^{\prime}, \nabla_{x^{\prime}} x^{\prime}\right)
$$

and $\nabla_{x^{\prime}} \nabla_{x^{\prime}} x^{\prime} / k c\left(1-c^{2}\right) \kappa$ is a unit vector field along $C$. Therefore, $\left\{e_{1}, e_{2}\right\}$ and hence parallel vector fields $\left\{E_{1}, E_{2}\right\}$ are orthonormal. Then $\{(x(\sigma)$, $y(\sigma))\}$ defined by (3.3) satisfies (2.5) 2 . By the differential equation (3.1) we have

$$
\left(\nabla_{x^{\prime}} x^{\prime}\right)(\sigma) /\left(1-c^{2}\right) \kappa=\cos k c \sigma \cdot E_{1}(\sigma)+\sin k c \sigma \cdot E_{2}(\sigma) .
$$


By $(3.3)$ and $\nabla_{x^{\prime}} y / c=-\sin (c \sigma+\gamma) E_{1}+\cos (c \sigma+\gamma) E_{2}$, we obtain

$$
\begin{aligned}
\nabla_{x^{\prime}} x^{\prime}=\left(1-c^{2}\right) \kappa & \cos [(1-k) c \sigma+\gamma] y \\
& -\left(1-c^{2}\right) \kappa \sin [(1-k) c \sigma+\gamma] \nabla_{x^{\prime}} y / c
\end{aligned}
$$

We put $a=g\left(x^{\prime}, y\right)$ and $b=g\left(x^{\prime}, \nabla_{x^{\prime}} y\right)$. By (3.4) and $\nabla_{x^{\prime}} \nabla_{x^{\prime}} y=-c^{2} y$, we obtain

$$
\begin{aligned}
a^{\prime} & =\left(1-c^{2}\right) \kappa \cos [(1-k) c \sigma+\gamma]+b \\
b^{\prime} & =-c\left(1-c^{2}\right) \kappa \sin [(1-k) c \sigma+\gamma]-c^{2} a
\end{aligned}
$$

Solving the above with $a(0)=g\left(x^{\prime}(0), y(0)\right)=-\left[\left(1-c^{2}\right) \kappa / k c\right] \sin \gamma$. we get

$$
\begin{gathered}
a=-\left[\left(1-c^{2}\right) \kappa / k c\right] \sin [(1-k) c \sigma+\gamma], \\
b=-\left[\left(1-c^{2}\right) \kappa / k\right] \cos [(1-k) c \sigma+\gamma] .
\end{gathered}
$$

So, (3.4) is rewritten as $\nabla_{x^{\prime}} x^{\prime}=-k b y+k a \nabla_{x^{\prime}} y$.

\section{$\S 4$. Proof of Theorem A}

Assume that a Killing vector field $Z$ on $\left(T_{1} M(k), g^{S}\right)$ is not fiber preserving. Let $\left\{\phi_{t}\right\}$ be a (local) 1-parameter group of local isometries generated by $Z$. Since each fiber is totally geodesic in $\left(T_{1} M(k), g^{S}\right)$ and isometric to a unit $(m-1)$-sphere, we can choose a great circle $\tilde{C}=\{(x(\sigma), y(\sigma)) ; 0 \leq$ $\sigma \leq 2 \pi\}$ of length $2 \pi$ in a fiber, such that $\phi_{t} \tilde{C}$ is not contained in a fiber for $t$ with $0<t<\varepsilon$. Here we can assume that the domain of definition of $\phi_{t}$ contains the fiber containing $\tilde{C}$. In this case, for small $t$ with $0<t<\varepsilon$, $C_{t}=\pi \phi_{t} \tilde{C}$ is a small closed curve, and it can not be a geodesic in $(M(k), g)$. We have $0<\left|c_{t}\right|<1$. By Theorem C, (ii-b-1), and (2.9) we see that $k \neq 0$ and $\alpha_{t} \neq 0$. By (ii-c) we see that the angle $\theta_{t}(\sigma)$ between $y_{t}(\sigma)$ and $C_{t}$ is given by

$$
\cos \theta_{t}(\sigma)=\left[\alpha_{t} / \sqrt{1-c_{t}^{2}}\right] \sin \left[(1-k) c_{t} \sigma+\beta_{t}\right]
$$

As $t \rightarrow 0$, we have $\left|c_{t}\right| \rightarrow 1$. So we have many small $t$ such that $(1-k) c_{t}$ is not an integer. This means $\theta_{t}(\sigma) \neq \theta_{t}(\sigma+2 \pi)$ for such $t$. This is a contradiction to the fact that $\tilde{C}_{t}$ is a closed geodesic for any $t$. 


\section{REFERENCES}

[1] P. Dombrowski, On the geometry of the tangent bundle, J. reine angew. Math., 210 (1962), 73-88.

[2] W. Klingenberg and S. Sasaki, On the tangent sphere bundle of a 2-sphere, Tôhoku Math. J., 27 (1975), 49-56.

[3] T. Konno, Killing vector fields on tangent sphere bundles, Kodai Math. J., 21 (1998), 61-72.

[4] P. T. Nagy, Geodesics on the tangent sphere bundle of a Riemannian manifold, Geom. Dedicata, 7 (1978), 233-243.

[5] S. Sasaki, On the differential geometry of tangent bundles of Riemannian manifolds I, Tôhoku Math. J., 10, (1958), 338-354, II, ibid., 14 (1962), 146-155.

[6] S. Tanno, Killing vectors and geodesic flow vectors on tangent bundles, J. reine angew. Math., 282 (1976), 162-171.

Tatsuo Konno

Mathematical Institute

Tôhoku University

Aoba-ku Sendai 980-8578

Japan

94m05@math.tohoku.ac.jp

Shukichi Tanno

Department of Mathematics

Tokyo Institute of Technology

Meguro-ku Tokyo 152-8551

Japan 